\title{
Ley de la conservación de la energía
}

Energy conservation law

\author{
Yuliana Vázquez-Arteaga ${ }^{a}$, Elizabeth Pérez-León ${ }^{b}$, Leonel Rojas-Juárez ${ }^{c}$, Jesús A. Ancelmo- \\ Ramírez $^{d}$
}

\begin{abstract}
:
Throughout history we have seen several process changes and it has been thought that this energy that processes have is eliminated, but what actually happens in the process is the so-called conservation of energy, that is to say that "energy it is not created or destroyed it is only transformed ", this means that the energy will never disappear, it will only become the objective that we have to carry out, where we will see a change in the process.

So now we know what happens to all the energies that produce the different types of processes when doing something. Example of the energy of process 1 there is a change and a process 2 is obtained, in these processes there is an energy transformation where energy is never lost, it is only transformed in process 2 .
\end{abstract}

\section{Keywords:}

Energy, transformer, process

\section{Resumen:}

En lo largo de la historia hemos visto varios cambios de procesos y se ha llegado a pensar que esa energía que tienen los procesos se elimina, pero lo que en verdad sucede en el proceso es la llama conservación de la energía es decir que "la energía no se crea ni se destruye solo se transforma", esto quiere decir que la energía jamás va a desaparecer solo se va a transformar en el objetivo que tengamos que realizar donde se verá un cambio en el proceso.

Entonces ahora se sabe que es lo que sucede con todas las energías que producen los distintos tipos de procesos en el momento de realizar algo. Ejemplo de la energía del proceso 1 hay un cambio y se obtiene un proceso 2, en dichos procesos hay una transf ormación de energía donde jamás se pierde la energía solo se transforma en el proceso 2.

\section{Palabras Clave:}

Energía, transformar, proceso

\section{Introducción}

La conservación de la energía empieza desde 1847 cuando el físico James Prescott joule enuncia el principio de la conservación de la energía que dice "LA ENERGIA NO SE CREA NI SE DESTRUYE SOLO SE
TRANSFORMA". Esto quiere decir que la energía se transforma de una forma a otra pero la cantidad total de la energía siempre permanece constante.

Existen diversos tipos de sistemas en los cuales podemos encontrar diferentes tipos de energías tales como la energía interna (asociada a la estructura interna de un

\footnotetext{
Yuliana Vázquez Arteaga, Universidad Autónoma del Estado de Hidalgo, https://orcid.org/0000-0002-2058-7975, Email: va338136@uaeh.edu.mx

b Elizabeth Pérez León, Universidad Autónoma del Estado de Hidalgo, https://orcid.org/0000-0002-2058-7975, Email: pe340684@uaeh.edu.mx

Leonel Rojas Juárez, Universidad Autónoma del Estado de Hidalgo, https://orcid.org/0000-0003-3443-6614, Email: ro383059@uaeh.edu.mx

d Jesús Artemio Ancelmo Ramírez, Universidad Autónoma del Estado de Hidalgo, https://orcid.org/0000-0002-6750-8939, Email: an341082@uaeh.edu.mx
} 
cuerpo), la química (que se pone en juego al producirse reacciones químicas), la eléctrica, la magnética, la mecánica, la elástica, la radiante, la gravitatoria, la sonora, la electromagnética y la nuclear [1].
Cuando la energía que se encuentra en un sistema pasa de una forma a otra, esto es la ley de conservación de la energía esta ley no tiene demostración matemática, es un Principio y se admite como tal [2]. 


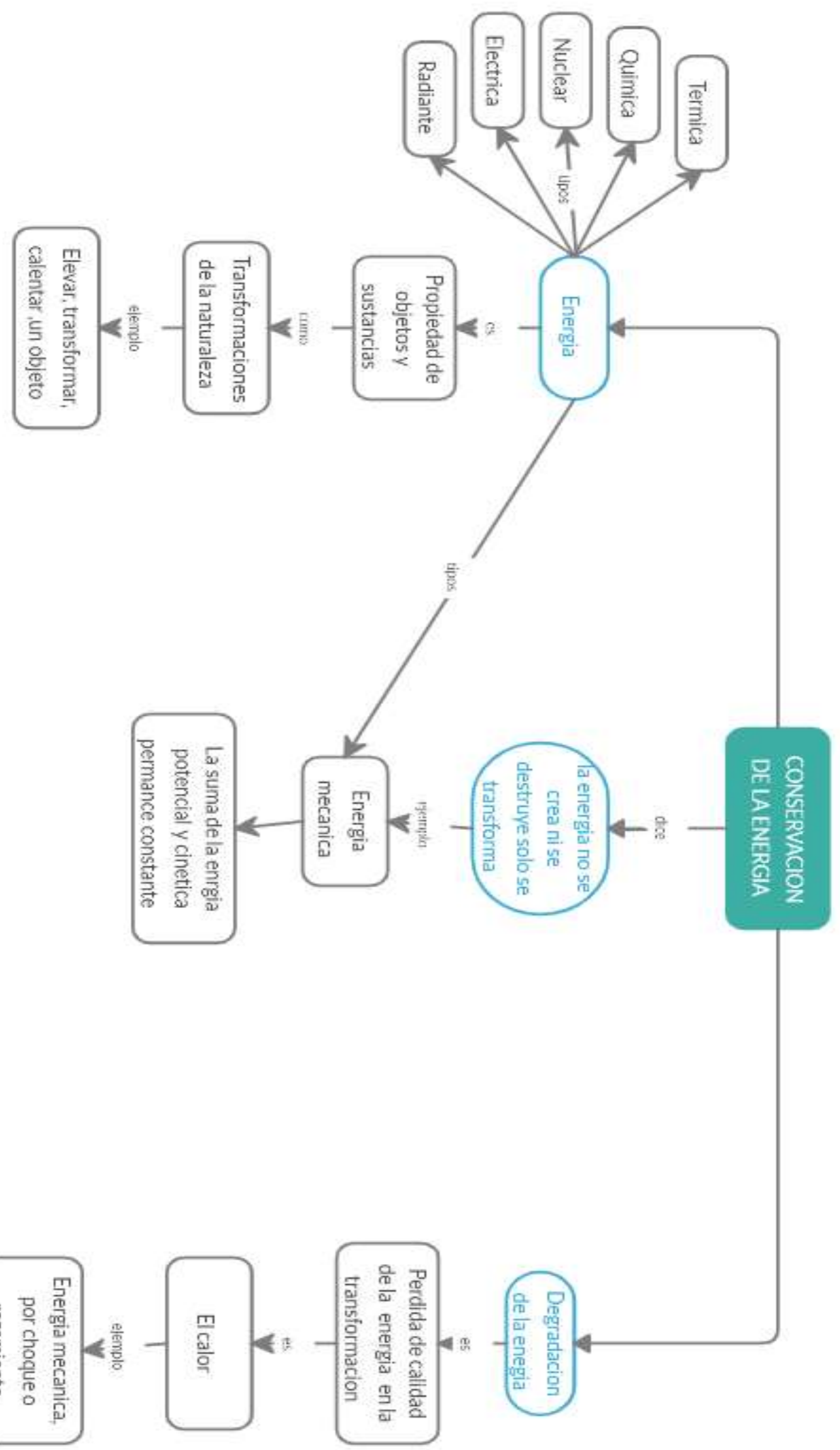




\section{Referencias}

[1] Tipler, P., \& Mosca, G. (2005). La conservación de la energía. En Física para la ciencia y la tecnología (págs. 178-192). Barcelona : Reverté.

[2] Medina Domínguez, A., \& Ovejero Sán, J. (11 de 2010). Física I. Obtenido de conservacion de la energía: https://www.academia.edu/13393717/3_aptrabyener1011

[3] Tippens, P. (2011). Conservación de la Energía. En Física conceptos y aplicaciones (págs. 157-171). Santa Fe: McGrawHitl.

[4] recio miraño, J. (2012). newton. Recupera do el 14 de marzo de 2020, de

la energia:

http://newton.cnice.mec.es/materiales_didacticos/energia/conservacion.ht $\mathrm{m} ? 3 \& 1$ 